\title{
AGENCIA Y POLIVICTIMIZACIÓN EN INFANCIA MIGRANTE: ANALIZANDO PERCEPCIONES PROFESIONALES
}

\section{AGENCY AND POLY-VICTIMIZATION IN MIGRANT CHILDHOOD: ANALYZING PROFESSIONAL PERCEPTIONS}

Iskra Pavez-Soto**, Daniela Poblete-Godoy ${ }^{\star \star *}$ y Carmen Alfaro-Contreras ${ }^{\star \star \star *}$

Resumen: El propósito principal de este artículo es revisar y comparar los conceptos de polivictimización y agencia de la infancia migrante. La noción de agencia remite al clásico debate teórico entre agencia y estructura dentro de las ciencias sociales introduciendo distinciones conceptuales y analíticas entre las diversas formas de agencia infantil en los procesos migratorios. Mediante el método cualitativo, se realizaron entrevistas semiestructuradas

* En este artículo se presentan resultados del Proyecto FONDECYT $\mathrm{N}^{\circ}$ 1170947 titulado "Múltiples violencias que afectan a las niñas y los niños migrantes en Chile: derechos, intervenciones sociales y políticas públicas” financiado por la Agencia Nacional de Investigación y Desarrollo de Chile (ANID).

** Dirección: General Gana 1702, Santiago de Chile, código postal: 8340000. Centro de Investigación en Educación (CIE), Universidad Bernardo O’Higgins. Iskra.pavez@ubo.cl

*** 08193 Bellaterra (Cerdanyola del Vallès), Barcelona. Doctoranda en Sociología, Universidad Autónoma de Barcelona, Becas Chile ANID. daniela. poblete@e-campus.uab.cat

**** General Gana 1702, Santiago de Chile. código postal: 8340000, Escuela de Tecnología Médica, Universidad Bernardo O’Higgins. carmen.alfaro@ubo.cl 
a profesionales del área de los servicios sociales, educación y salud, para conocer su percepción sobre la agencia infantil. Se concluye que las percepciones profesionales sobre la agencia infantil migrante se inscriben en perspectivas reduccionistas, que, desde la sumisión a la agresión, se basan en visiones culturalistas y estereotipadas en torno a la edad, el sexo-género o la nacionalidad. Por este motivo, urge visibilizar la agencia infantil libre de juicios estereotipados.

Palabras claves: agencia infantil; polivictimización; infancia migrante; percepción profesional.

Abstract: The main aim of this article is to review and compare the concepts of poly-victimization and agency of migrant childhood. The notion of agency refers to the classic theoretical debate between agency and structure within the social sciences, introducing conceptual and analytical distinctions between different forms of child agency in migratory processes. Using the qualitative method, semistructured interviews were conducted with professionals in the areas of education, health, and social intervention with the purpose of finding out their perception of children's agency. It is concluded that professional perceptions about migrant children's agency are inscribed in reductionist perspectives, which - from submission to aggression-are based on culturalist and stereotypical views on age, sex-gender or nationality. For this reason, it is urgent to study the children's agency without judgments and stereotypes.

Key words: child agency; poly-victimization; migrant childhood; professional perception.

\section{INTRODUCCIÓN}

La agencia de las personas migrantes es un tema que comienza a ser tratado en los estudios migratorios (Güell et al., 2020; Ródenas, 2017) y en los estudios de infancia (Pavez-Soto y Sepúlveda, 2019). De modo particular, este trabajo indaga sobre las estrategias de agencia que desarrollan las niñas y los niños migrantes, pero analizadas desde el punto de vista de técnicos y profesionales de servicios sociales, salud y educación, tomando el caso de Chile y considerando el sostenido aumento de los flujos migratorios durante los últimos años. En concreto nos preguntamos: ¿Cuáles son las nociones de agencia 
infantil que subyacen a los discursos expertos que atienden a la niñez migrante? Se esboza como respuesta una hipótesis referida a los paradigmas del saber experto, los cuales conceptualizan a la niñez migrante como un sujeto en falta o en déficit (Pavez-Soto et al., 2020).

La población infantil representaría en torno al 14\% de la población migrante total y proviene mayormente de países latinoamericanos como Venezuela $(30,5 \%)$, Perú $(15,8 \%)$ y Haití $(12,5 \%)$ (INE y DEM, 2020). En Chile, algunas investigaciones ya han evidenciado las dificultades en los centros educativos para enfrentar las situaciones de racismo, discriminación y acoso que padece la niñez migrante (Cerón, Pérez Alvarado y Poblete, 2017; Pavez-Soto et al., 2019; Poblete y Galaz, 2017; Riedemann y Stefoni, 2015; Tijoux-Merino, 2013). Si bien un $71 \%$ de las niñas y niños migrantes ha reconocido haber sufrido algún tipo de discriminación (Ministerio de Desarrollo Social 2020), se debe aclarar que lamentablemente los malos tratos también ocurren en la infancia local (Consejo Nacional de la Infancia, 2018; UNICEF, 2012). Sin embargo, para la niñez migrante la discriminación se agudiza porque confluye con otros mecanismos de discriminación, por ejemplo, en el acceso a la vivienda (Margarit y Bijit, 2014), lo cual desemboca en situaciones de exclusión social, se observa mayor hacinamiento en los hogares de jefatura extranjera $(20,6 \%)$ en comparación con nacionales $(5,8 \%)$ (Ministerio de Desarrollo Social, 2017).

Anteriormente, habíamos estudiado los mecanismos de agencia infantil para enfrentar la discriminación en el ámbito escolar y en la violencia intrafamiliar (Pavez-Soto 2011, 2018). Sin embargo, hasta ahora pocas investigaciones (Cortez et al., 2019; Galaz et al., 2019) han conjugado la capacidad de agencia de las niñas y los niños migrantes cuando padecen distintos tipos de violencia de modo simultáneo. Aún resta trabajo por discutir hasta qué punto niñas y niños expuestos a procesos altamente hostiles son capaces de ejercer su capacidad de agencia, sin el riesgo de profundizar o repetir las experiencias de victimización que han vivido. En otras palabras, hasta qué punto la estructura social determina las posibilidades de ejercer la agencia. El artículo también ofrece resultados empíricos de primera fuente con el objetivo de explicar cómo la mirada profesional interpreta las expresiones de agencia infantil frente a distintas experiencias de victimización.

La estructura del artículo es la siguiente: en primer lugar, se presentan los aportes del concepto de polivictimización aplicado al 
campo de las migraciones internacionales. Por otra parte, desde la sociología de la infancia actualizamos el debate teórico y revisamos cuáles son los mecanismos de agencia que niñas y niños migrantes han desarrollado para enfrentar el proceso migratorio en una diversidad de contextos. Luego se presentan los resultados de la investigación a partir de un análisis de entrevistas a profesionales de servicios sociales, educación y salud. Se finaliza con una discusión y conclusiones preliminares en torno a los desafíos de investigación futura.

\section{TENSIONES TEÓRICAS ENTRE POLIVICTIMIZACIÓN Y AGENCIA INFANTIL}

El problema de investigación se inscribe en la necesidad de analizar las nociones de agencia que subyacen en técnicos del área de los servicios sociales, la educación y la salud en los procesos de intervención profesional con niñas y niños migrantes que enfrentan diversas situaciones de violencia simultáneamente (polivictimización). De modo particular, se toma el caso de Chile, por el importante aumento de la población extranjera y el respectivo incremento de demanda de prestaciones y atención, lo cual ofrece la oportunidad de revisar los paradigmas que sostienen la acción pública en situaciones altamente complejas.

Se debe tener en cuenta que la organización de los servicios sociales, educación y salud en Chile se rige por un modelo mixto en el cual los distintos prestadores de servicios no están necesariamente articulados entre sí porque dependen de distintas administraciones municipales, ministeriales o privadas con diversos grados de autonomía y poder financiero, lo cual probablemente condiciona la utilización de ciertos paradigmas y/o incide en los niveles de la intervención.

Para entrar en materia es preciso definir el concepto de polivictimización, el cual alude a la exposición a múltiples formas de violencia, lo cual desencadena procesos denominados como victimización. Habitualmente, se tendía a exacerbar los problemas de salud asociados a un solo tipo de victimización y sus eventuales riesgos futuros. Las primeras definiciones argumentan que la victimización depende de varios factores, ya sea ambientales, 
familiares o personales; por lo tanto, la idea de polivictimización sugiere que no se trata solo de eventos de violencia múltiple, sino, además, la polivictimización podría representar una condición (Finkelhor et al., 2007).

Las investigaciones concuerdan en que los niños y las niñas que han sido victimizados previamente tienen un mayor riesgo de sufrir polivictimización posterior, mayores probabilidades de experimentar impactos psicobiológicos menos reversibles en el tiempo y mayores niveles de ansiedad, unido a otra sintomatología traumática (Cedeño, Martínez-Arias, y Bueno, 2014; Finkelhor et al., 2011; Finkelhor, Ormrod, y Turner, 2007; Pereda y GallardoPujol, 2014). De acuerdo a Pereda y Gallardo (2014), las polivíctimas tendrían dificultades en la conformación de relaciones que la sociedad espera o que se consideran relevantes y satisfactorias para la vida moderna, lo cual tendría implicancias en la autoestima y la confianza personal. Si bien se ha registrado un nivel de caracterización del fenómeno, por ejemplo que es más común en niños mayores (Finkelhor et al., 2007, 2011) y que puede afectar más a las niñas (Finkelhor, 2011), Pereda y Gallardo (2014) aún visualizan desafíos de investigación, por ejemplo: identificar cuáles son las manifestaciones en la polivictimización según diferentes edades o por sexo-género; así como ampliar estos estudios a países con distintos contextos socioculturales, pues podrían dar indicaciones sobre la influencia de las diversas dimensiones culturales y entender las razones de las diferencias observadas. No obstante, los desafíos pendientes, se debe señalar que en Latinoamérica los estudios sobre polivictimización son escasos (Cortez et al., 2019). Además, se reconoce una inconsistencia de los datos entregados por los organismos oficiales de la infancia y organismos internacionales (Cortez, Beltrán, y Jara, 2017). Por lo tanto, en esta región existe adicionalmente la necesidad de realizar estudios y una integración de la producción académica y la práctica profesional que permita abordar la victimización (Cortez et al., 2017).

En este contexto cuyo alcance ya es acotado, investigar las vivencias de polivictimización en personas migrantes ha sido menos explorado aún. Por ejemplo, se han estudiado a las personas latinas en España como potenciales polivíctimas y el consecuente desarrollo de una constelación de psicopatologías duraderas que impactan en el rendimiento escolar, en el nivel de las competencias laborales y finalmente en las posibilidades de vivir en condiciones de exclusión 
social (Cedeño et al., 2014); así también se ha encontrado evidencia estadísticamente significativa que asocian victimización múltiple y afecciones en salud mental más alta para adolescentes migrantes en Cataluña en comparación con sus pares locales (Segura et al., 2015); y también sobre mujeres inmigrantes en Portugal, donde se menciona la influencia no solo de factores individuales e interpersonales, sino también estructurales y sociales, lo que ha llevado a las investigadoras a cuestionarse las implicaciones para la intervención y el empoderamiento de estas mujeres (Gonçalves y Matos, 2020).

Estos resultados, así como la conceptualización de polivictimización, originarias de las disciplinas de la salud mental, sugieren una condición e impacto que las víctimas cargarían a lo largo de su vida (Ford y Delker, 2018). Sin embargo, este argumento entra seriamente en tensión si se introducen variables estructurales (históricas, económicas y culturales) y si se analiza la experiencia de los niños y las niñas migrantes desde perspectivas críticas, como la sociología de la infancia (Pavez-Soto y Sepúlveda, 2019).

Lo anterior remite al clásico debate dentro de las ciencias sociales sobre la relación entre acción y estructura social. Uno de los referentes en esta visión teórica es Talcott Parsons (1968, 1999). De acuerdo a James y Prout (1997) niños y niñas producen y negocian junto a otros actores un marco estructural de la infancia en la sociedad. Por lo tanto, son agentes sociales activos, capaces de actuar de acuerdo a un determinado contexto (James y Prout, 1997).

Ahora bien, es preciso declarar que el contexto estructural deja un marco de acción minoritario a los niños y las niñas (Mayall, 2002). Existe la tendencia que la acción infantil no sea reconocida mientras se argumenta un orden natural en base a la edad. Bajo este supuesto, la edad es una condición que define competencias y capacidad de acción, que genera relaciones de poder (Qvortrup, 1994). Según Mayall (2000) bajo la suposición que la niñez sería solo un objeto resultado de la socialización adulta, por lo tanto, despliegan acciones desde un lugar subordinado. Escasamente niñas y niños son reconocidos como actores independientes en los espacios públicos o se visualiza la negociación permanente con la acción adulta (Mayall, 2000).

La infancia es un constructo social y cultural, un fenómeno histórico y complejo, pues cambia en el tiempo y de acuerdo a su ubicación geográfica (Haring, Sorin, y Caltabiano, 2019; James y Prout, 1997; Jenks, 1996). En este sentido, el reconocimiento de la 
agencia infantil representa un cambio de paradigma (Garnier, 2015; Haring et al., 2019) pues se distancia de enfoques clínicos y biológicos que comprenden a niños y niñas como personas incompletas o seres respondientes. El reconocimiento de la agencia infantil es un cambio paradigmático que da validez a las voces infantiles, lo que significa reconocerlos como actores sociales y no como meros objetos de protección o cuidado.

Sin embargo, tal como las investigaciones sobre polivictimización se encuentran en pleno avance, la conceptualización en torno a la agencia infantil también está en una etapa de construcción. Según Strandell (2010, p. 177) la división acción-estructura se encuentra en una etapa inicial en los estudios de la sociología de la infancia, con algunas ideas que se inclinan más hacia la dimensión estructural y otras más orientadas a la acción. El problema resulta más evidente cuando la experiencia infantil se ubica en los márgenes de las definiciones modernas de los derechos infantiles, considerando que en nombre del interés superior infantil se justifica la restricción de ciertos derechos, especialmente aquellos relativos a la participación.

En palabras de Bordonaro y Payne (2012), el concepto de agencia también nos enfrenta a los dilemas ético-morales de intervención cuando la agencia infantil no ha tenido condiciones suficientes para expresarse a través de un proceso autorreflexivo libre, sino que se traduce sin más alternativas y por necesidad, en reacciones que atentan contra la propia seguridad infantil y los arriesga a ser catalogados como desviados por el orden social y moral existente. Thompson et al. (2019) señalan que la percepción de la agencia infantil transita entre enfoques binarios. Según Ungruhe (2019) oscilan entre la dicotomía de la romantización o la victimización. De acuerdo a este investigador, especializado en estudios de infancia en África, autores proponen la idea de graduación de la agencia infantil para capturar las estrategias de afrontamiento en entornos de alta marginación social. En contraposición a la agencia "gruesa", o sea cuando los márgenes para la acción infantil son más amplios, se categorizan agencias "restringidas", "delgadas" o "limitadas" (Klocker, 2007). Es decir, cuando los contextos son excesivamente restrictivos y no hay grandes posibilidades para tomar decisiones. En estos casos, también se usan otras categorías como, por ejemplo:

- Agencias "tácticas" (Honwana, 2005) cuando niños y niñas hacen frente a las circunstancias inmediatas que les afectan 
y no tendrían posibilidad de ser plenamente conscientes de los objetivos de sus acciones, ni de anticipar los beneficios al largo plazo;

- Agencias de "protección" (Atkinson-Sheppard, 2017) reúne a las agencias tácticas (Honwana, 2005) y delgadas (Klocker, 2007) con un componente adicional: desarrollan acciones a cambio de la protección que no pueden obtener de otra manera;

- Agencias "ambiguas" (Bordonaro y Payne, 2012) para referirse a aquellas expresiones de agencia infantil que no se orientan hacia los objetivos consistentes con los ideales políticos de auto liberación, y que por esta razón terminan siendo moldeadas por el mundo adulto para que sean consistente con estándares morales y sociales específicos del "interés superior". En términos más simples, se refiere a aquella agencia ligera y típicamente tachada como desviación social;

- Agencias “autodestructivas” (Gigengack, 2008), es deciraquellas cuyas decisiones acarrean mayores riesgos de vulneración. Pavez-Soto y Sepúlveda (2019) citan a las reflexiones de Llobet (2012) respecto a las infancias en situación de calle, pandillas infantiles o pequeñas faltas que denominan como agencias negativas.

Para problematizar estas ideas, Strandell (2010) destaca la necesidad de aplicaciones más fluidas y dinámicas, Thompson et al. (2019) y Huijsmans (2012) proponen especialmente para el caso de los niños y niñas migrantes, un enfoque no binario de agencia que reconozca y abrace los derechos y vulnerabilidades, examinando sus resistencias, competencias, objetivos y fortalezas. Además, de tener en cuenta los factores sociales y relacionales que sustentan y dan forma a la toma de sus decisiones.

Aunque en otro campo de investigación distinto de donde surge el concepto de polivictimización, desde la vereda de la sociología y los estudios etnográficos se ha podido dar respuesta a uno de los desafíos que Finkelhor (2007) ha dejado pendientes: trabajar para identificar por qué las niñas y los niños se transforman en poli-víctimas, ya que hasta esa fecha se había explicado solo en términos del apego y la crianza, de la capacidad de autoprotección o desde una perspectiva criminológica. El investigador había expresado que era necesario comprobar modelos que analicen múltiples factores de riesgo para 
la poli-victimización, como son la resiliencia y vulnerabilidad. Sin embargo, como ya habíamos advertido, los enfoques de la agencia infantil colisionan con los hallazgos en el campo de la psicología, que consideran las experiencias de polivictimización como predictores de traumas, representando factores de riesgo futuro, aseverando incluso que las victimizaciones múltiples pueden ser señal de que las niñas y los niños están pobremente supervisadas o socialmente aisladas y, por tanto, serían "objetos" desprotegidos que presentan deficiencia en interacciones sociales o problemas psicológicos preexistentes (Finkelhor et al., 2007; Finkelhor, Ormrod, y Turner, 2009). Se ha hablado de una permanencia de los efectos, es decir, que sería difícil escapar de la polivictimización (Finkelhor et al. 2011).

Aún más, según Cedeño et al. (2014), se ha aceptado como un lugar común la premisa de la transmisión intergeneracional de la violencia, planteando un "esquema de victimización" entre padres e hijos/as que implica que las víctimas quedan vulnerables, incompetentes social y cognitivamente y, por tanto, más expuestos para ser abusados nuevamente. En la misma línea, autores como Guerra et al. (2017) señalan que, en casos de efectos postraumáticos se desconoce cómo se puede ayudar a prevenir la sintomatología de la polivictimización o cómo operaría el rol protector de la autoeficacia y apoyo social en estos casos. Bajo el supuesto que a mayor polivictimización, menor autoeficacia y mayor la sintomatología (Guerra et al., 2017).

\subsection{Antecedentes de agencia infantil en la migración internacional}

Como se dijo anteriormente, la investigación ha comenzado a centrarse en las experiencias que viven las niñas y los niños migrantes en los procesos migratorios. Metodologías focalizadas en la niñez a través de métodos etnográficos (Moscoso, 2013) y técnicas como entrevistas (Pavez-Soto, 2011, 2018) o dibujos infantiles (Dreby y Adkins, 2012) han logrado identificar cómo significan la experiencia migratoria de sus madres, su propia experiencia y también cómo significan sus vínculos familiares. Estas investigaciones han contribuido a visualizar la agencia infantil, es decir la capacidad de niños y niñas para incidir sobre las cosas que les afectan (James y James, 2012) 
Investigaciones a nivel internacional, en diversos contextos, dan cuenta de las estrategias que niños y niñas despliegan para incidir en los procesos migratorios que les afectan. Entre los resultados más notables de la agencia destacan una serie de mecanismos para definir y negociar la autoridad y las relaciones de parentesco de forma dinámica a lo largo de la trayectoria migratoria (Caneva, 2015; Moscoso, 2013; Pavez-Soto, 2011; Piras, 2016; Sime y Fox, 2015).

Por ejemplo, una investigación de familias latinoamericanas en Italia es ilustrativa (Caneva, 2015). De acuerdo a Caneva (2015) niñas y niños pueden decidir si desean reconocer el rol de sus madres y mediante qué mecanismos. La definición se relaciona primero con la actitud de los niños y niñas en el acercamiento a sus madres; segundo, con el compartir una vida, es decir, con un largo esfuerzo emocional de vincularse con sus madres a través de la distancia o una vez acaecida la reagrupación familiar. Las expectativas normativas sobre las relaciones madre-hija se ven socavadas por la negociación y flexibilidad que van poniendo en la relación. El tiempo de separación también influye en cómo se redefine la relación, a menos tiempo menor la dificultad y a mayor tiempo, más complicaciones se pueden observar, ya que al no haber convivido se deben esforzar en crear sensaciones de cercanía. Se ejemplifican dos casos donde las madres biológicas no mantuvieron contacto permanente ni se esforzaron en vincularse y una vez reunidas se inician disputas sobre afinidades y cercanías emocionales, se jerarquiza el vínculo con sus cuidadoras que permanecen en el país de origen por sobre el parentesco genético. Cuando las madres migran, por ejemplo, niños y niñas que ya han construido una biografía compartida con ellas, viven la separación como un acontecimiento triste e influye en las relaciones de parentesco que se construyen en un futuro. Una vez reunidos, niñas y niños tienen que reconstruir las relaciones familiares, y aprender a vivir de nuevo con su madre. Este proceso de negociación de las relaciones puede ocurrir lentamente, pero niños y niñas suelen tener éxito. En algunos casos, se mantiene la separación en contra de la voluntad de sus madres, al negarse a abandonar la relación con sus cuidadoras en el país de origen. La misma situación ya se había registrado en niños y niñas migrantes en Chile (Pavez-Soto, 2011) y de la niñez mexicana cuyas madres residían en Estados Unidos (Dreby, 2007) y mostraba desinterés o indiferencia al negarse a contestar sus llamadas o sus visitas; otro 
acto destacado era el pedir permiso a sus cuidadoras en México, como una forma de despreciar la autoridad materna o significar una autoridad ambigua e incluso guardar resentimiento hacia las madres, por no cumplirse las expectativas (Dreby, 2015).

También se han evidenciado expresiones relativas a la percepción de sentirse o situarse al margen del proyecto migratorio. De acuerdo a Dreby (2007) cuando las niñas, dada su condición de sexo-género, se quedan en el país de origen su capacidad de actuación se vuelve más difícil frente a situaciones complejas y acaban realizando diversos actos que podrían ser interpretados como expresiones de agencia y que tensionan la moralidad que recae sobre las niñas, tales como fuga del hogar, alcoholismo, drogadicción o abuso hacia niñas menores. Otra situación sería lo contrario: no quieren ocasionar problemas a sus madres, padres o cuidadoras, por lo que asumen esta responsabilidad de manera solitaria, lo cual aumenta su agresividad. Así también es posible que pierdan el interés por la escolarización y terminen desertando de la escuela. Entre las razones que explican la deserción es que cuando las madres han partido de México, las niñas experimentaron niveles de depresión, las profesoras reportan problemas de disciplina y situaciones de acoso entre pares. El sentimiento más común expresado fue la falta de apoyo académico de las cuidadoras en México, dado el bajo nivel educacional que éstas poseen. Las consecuencias negativas que pudieran experimentar niños y niñas que han quedado en el lugar de origen con frecuencia ha denominado "left-behind children", tendencia que no solo se ha registrado en la migración internacional, sino también en la migración interna (Chen, Liang y Ostertag, 2017; Hongwei et al., 2018).

Sin embargo, en el caso que el niño o niña migre, se observó un agenciamiento afirmativo de los propios derechos en el proyecto migratorio. La investigación de Thomson et al. (2019) sobre niñas centroamericanas y mexicanas en un centro de detención señala que ellas son capaces de expresar las motivaciones personales para la migración. Conclusiones similares se habían encontrado en los estudios sobre la infancia migrante en Chile (Pavez-Soto, 2011; Pavez-Soto et al., 2019). Este es un indicador de pensamiento reflexivo, que refleja la capacidad de construir o identificarse con un acto asociado a la búsqueda de la reunificación familiar y las respectivas oportunidades de educación y mejora. De igual forma, otra expresión corresponde al ejercicio activo de voluntad infantil o 
el reclamo de derechos. Se posicionan fuertemente en esta agencia cuando se ven en riesgo, están detenidas o embarazadas, para mitigar riesgos en el trayecto de migrar (Thompson et al., 2019). Otras expresiones afirmativas han sido registradas por Rübner (2016) en un estudio comparativo entre Inglaterra y España, señala que las jóvenes entrevistadas eran capaces de idear el proyecto migratorio, por ejemplo, en el contexto inglés la mayoría centraba su futuro en la educación o búsqueda de alguna ocupación; mientras que en Madrid, aunque tenían este mismo tipo de aspiraciones, dudaban del acceso a la educación universitaria, por lo tanto, optaban por formación técnica y trabajo al mismo tiempo (Rübner, 2016). En este contexto, una expresión de agencia común en la migración es la mantención de los lazos familiares y las redes transnacionales a través de la tecnología (Rübner, 2016; Sime y Fox, 2015).

Por último, también se han identificado expresiones que se podrían catalogar como tácticas. En el caso de las niñas mexicanas en centros de detención, existen expresiones de agencia menos abiertas o que coocurren con las acciones afirmativas, como, por ejemplo, divulgar o repetir información de las policías u otras niñas (Thompson et al., 2019). Si bien en dicho estudio se analiza a niños y niñas, se toma solo el caso de las niñas como un ejemplo de agencia táctica. Thompson et al. (2019) reporta un aumento de la vulnerabilidad que supone angustia emocional al no poder lograr sus cometidos de migrar, producto de las detenciones policiales. Conclusiones similares han sido encontradas en el caso de la niñez refugiada en Alemania (Wihstutz, 2020).

\section{METODOLOGía}

La metodología de esta investigación fue de carácter cualitativo. Se realizaron entrevistas semiestructuradas a 40 profesionales de nacionalidad chilena y que trabajan en instituciones públicas y organizaciones no gubernamentales en el área de servicios sociales, educación y salud.

La pauta de entrevista se orientó a develar los paradigmas de la infancia con los cuales ejercen su profesión, respecto a diferentes ejes de diferenciación como es el sexo-género, el estatus migratorio, el territorio de residencia, la edad y la nacionalidad. Debido a las 
restricciones de acceso a las instituciones y organizaciones, como es la alta carga laboral, los criterios de selección de la muestra fueron dos: profesionales que hayan trabajado con infancia migrante y que residieran en alguna de las cuatro regiones del país con mayor presencia de población migrante: Metropolitana, Antofagasta, Tarapacá y Valparaíso (Instituto Nacional de Estadísticas, 2018). Se entrevistó a 10 profesionales por región, 31 de éstas fueron mujeres, 38 de nacionalidad chilena, con una media de 39 años de edad. Todas las aplicaciones garantizaron el anonimato, el derecho a la información y el respeto a los participantes a través de la aplicación de un protocolo ético y la utilización de Formularios de Consentimiento y Asentimiento Informado.

Las nacionalidades de niños y niñas que aparecen en las entrevistas de profesionales corresponden a flujos migratorios establecidos en Chile, como es la nacionalidad peruana y la boliviana, países con los cuales se comparte frontera al norte de Chile y donde también son habituales los flujos circulares (Parella y Tapia, 2015) con estancias mensuales en el caso de personas trabajadoras. También fueron referenciados flujos migratorios recientes, como son la nacionalidad colombiana y haitiana, siendo esta última la única no castellano hablante en la investigación. Cabe mencionar que, en el corpus textual analizado, las y los profesionales no hacen distinción entre niños/as que han migrado a Chile o que son descendientes de madre o padre extranjero/a (con nacionalidad chilena por haber nacido en Chile) y que se han socializado en este país; cuestión que, por el contrario, fue distinguida por niños, niñas o madres de origen extranjero (Pavez-Soto et al., 2020).

Las entrevistas fueron categorizadas mediante el uso del programa informático Atlas.Ti. Si bien la finalidad del análisis no ha sido crear teoría fundamentada en datos, se utilizaron dos tipos de codificación originarias de la Teoría Fundamentada a modo de herramientas analíticas útiles para gestionar, ordenar y relacionar datos cualitativos. Se entiende la codificación como el procedimiento de abstracción y agrupación de conceptos según sus propiedades y dimensiones sobresalientes (Strauss \& Corbin, 2002, pp. 73-74). De este modo cada código corresponde a una etiqueta nominativa que agrupa un conjunto de citas textuales de las entrevistas. En primer lugar, se realizó un procedimiento de codificación abierta de citas con el objetivo de explorar los datos, identificando conceptos claves, sus propiedades y dimensiones, 
vale decir las características de los conceptos, sus significados y escalas de variación (Strauss \& Corbin, 2002, p. 110). Mediante la comparación continua de los datos (Strauss \& Corbin, 2002, p. 148) se logró identificar relaciones y contradicciones entre conceptos. La comparación permitió densificar el análisis con nuevos subcódigos mediante el procedimiento de codificación axial que consiste en relacionar subcategorías conceptuales de acuerdo a la variación de las propiedades y dimensiones de cada concepto (Strauss \& Corbin, 2002, p. 134). De esta forma se subcodificaron las citas y se agruparon en familias de códigos. A partir de ello se creó un mapa semántico que grafica las relaciones entre conceptos, así como su comparación, orden y jerarquía que orienta la presentación y redacción de los resultados.

\section{RESULTADOS}

Las expresiones de agencia infantil de las niñas y los niños migrantes en Chile están sujetas a valoraciones desde el punto de vista adulto, así como también incluyen explicaciones respecto a sus causas.

\subsection{Agencia pasiva}

La siguiente cita es elocuente para graficar las formas en que niñas y niños responderían a la violencia se encuentran mediadas fundamentalmente por la edad, el sexo-género, las características fenotípicas o la nacionalidad. Interpretaciones de este tipo podrían incluir algún estereotipo racista asociado a la seguridad, puesto que se transita entre dos polos de clasificación: la infancia que debe ser protegida y la infancia de la cual habrá que proteger a la sociedad (Sellenet, 2007; Gaitán y Liebel, 2011).

Hay dos posturas, una cuando el niño es pequeñito da lástima, te dan ganas como de alimentarlo, de protegerlo, de buscarlo, pero cuando este niño crece y se vuelve adolescente, causa miedo, porque lo asocias a un estereotipo que nos enseñaron que es el... como dicen "el negro ladrón" o que te puede causar más daño, que a lo mejor el flaite chilensis (...) Y sí, hay un trato distinto, pero es algo, 
como, que viene del subconsciente a causa de estos estereotipos que manejamos (trabajadora social, institución pública, I región de Tarapacá, Iquique).

Según estas observaciones, algunas niñas y niños no lograrían ver que están siendo violentadas, aceptarían las agresiones y no buscarían ayuda en las personas adultas, una situación pasiva en contraste con quienes responderían a las agresiones, lo cual se podría interpretar como agencia reactiva.

Ellos no se dan cuenta que están sufriendo violencia, es cuando llegan al programa, es cuando se dan cuenta que un grito no es una forma de hablar (...), el que tu profesor te grite u ordene fuertemente "oye, ordena tu silla" o algo así, tampoco lo ven como algo malo, porque es el mismo trato que reciben en sus casas (trabajadora social, institución pública, I región de Tarapacá, Iquique).

A su vez, estas clasificaciones también polares, se asocian a nacionalidades en particular, lo cual arroja evidencia de xenofobia y visiones estereotipadas sobre determinados colectivos, generalizando actitudes puntuales sobre un grupo humano:

Las conductas de, principalmente, de la gente de Colombia, son mucho más agresivos, su contexto es de violencia, de insulto, en comparación con los bolivianos y los peruanos, que son mucho más sumisos (profesora, escuela pública, II región de Antofagasta).

\subsection{Agencia activa}

También es posible ver otro conjunto de discursos profesionales acerca de niñas y niños migrantes que realizan tareas que podrían verse como un trabajo y que reciben algo a cambio (dinero, especies, servicios, etc.) junto a sus madres y padres o, que en sus hogares asumen labores domésticas o de cuidado de otras personas (niñas más pequeñas o mayores). Además, se incluye aquí a quienes de forma eventual o simultánea a las tareas que desarrollan son víctimas de agresiones en su entorno. Estas experiencias son calificadas como niñas y niños "marentalizadas o parentalizadas", es decir que, desde el paradigma dominante de la infancia moderna (Gaitán y Liebel 2011) desarrollan labores que no corresponden a 
su nivel de desarrollo y que deberían asumir las personas adultas cuidadoras, quienes serían vistas como figuras ausentes, que no protegen de la violencia, costumbre que se asociaría a ciertas culturas o nacionalidades, reificando una visión culturalista.

Los padres no se involucran más allá. No se involucran más allá... sí, es difícil eso, porque al final, están bien solos los niños en la escuela (terapeuta ocupacional, institución de salud, Región Metropolitana, Santiago).

Cuando se trata de familias no castellano hablantes, tendrían dificultades para involucrarse en las situaciones de discriminación que vivirían niños y niñas en las escuelas, pero dicha hostilidad también la sufrirían las personas adultas, porque está motivada por el racismo:

Los papás van a las reuniones de apoderados, pero no entienden nada (...) En la reunión de apoderados una mamá, así: 'ay, estos negros no hablan (...) negro culiao [sic]'... cachai, ese es el apelativo (abogada, ONG, Región Metropolitana, Santiago).

Frente a estas situaciones, los y las profesionales identifican que su rol, en tanto personas adultas, es proteger y dar acogida emocional a la niñez migrante $y$, en algunos casos extremos, ofrecer asistencia material (ropa, zapatos, elementos escolares); necesidades que se asocian con estereotipos según cierta nacionalidad. De acuerdo a estas declaraciones, lo esperable en estos casos es que niños y niñas pidan ayuda a la profesional adulta y sea ella quien resuelva la situación ("acudir a un adulto"). Niños y niñas de menor edad cumplirían esta expectativa, sin embargo, cuanto más avanza la edad van resolviendo los conflictos de manera más autónoma y no estaría dentro de las expectativas deseables.

Constantemente se les recuerda que tienen que acudir a un adulto para comentar si tienen una situación conflictiva y que les ayude a que la resuelvan. Y suelen avisar normalmente, cuando van creciendo, normalmente los de séptimo y octavo, ya no tanto (psicóloga, escuela pública, Región Metropolitana, Santiago).

El análisis de datos cualitativos indica que existe un conjunto de discursos adultos que distinguen expresiones de agencia infantil que se expresan de modo pasivo y corresponden a las vivencias de las 
niñas y los niños que serían víctimas de vulneración de derechos (ver tabla 1). De acuerdo al discurso profesional, las víctimas tienden a normalizar la violencia, al retraimiento conductual y a la aceptación de las agresiones que reciben de parte de las personas cuidadoras o del grupo de pares, nacionales o migrantes.

\section{TABLA 1}

PERCEPCIONES ADULTAS PROFESIONALES DE LA AGENCIA INFANTIL MIGRANTE

\begin{tabular}{|l|l|l|}
\hline Categorías de análisis & \multicolumn{1}{|c|}{ Agencia infantil "pasiva" } & \multicolumn{1}{c|}{ Agencia infantil "activa" } \\
\hline Formas de expresión & $\begin{array}{l}\text { vulneraciones } \\
\text { normalización } \\
\text { aceptación } \\
\text { trabajo doméstico y cuidado }\end{array}$ & $\begin{array}{l}\text { defensa verbal y física entre pares } \\
\text { agrupamiento entre pares } \\
\text { conductas transgresoras }\end{array}$ \\
\hline Estereotipos & $\begin{array}{l}\text { sumisión } \\
\text { retraimiento } \\
\text { timidez } \\
\text { dependencia } \\
\text { idealización }\end{array}$ & $\begin{array}{l}\text { violencia } \\
\text { agresión } \\
\text { desadaptación } \\
\text { marentalización /parentalización }\end{array}$ \\
\hline Rol adulto & madres ausentes & madres intervienen por su cuenta \\
\hline Respuesta profesional & $\begin{array}{l}\text { acogida emocional } \\
\text { asistencia material } \\
\text { protección } \\
\text { culturalización }\end{array}$ & $\begin{array}{l}\text { estigmatización } \\
\text { sexismo } \\
\text { determinismo de la trayectoria vital } \\
\text { responsabilización infantil }\end{array}$ \\
\hline
\end{tabular}

Fuente: elaboración propia.

Vale la pena evidenciar el sesgo de sexo-género implícito que aparecería latente en una idea normativa de familia migrante, donde la mujer sería la principal responsable del cuidado infantil. Es posible observar en algunos discursos profesionales que esta condición cultural o división sexual del trabajo parece naturalizarse, lo que confirma la necesidad de revisión de los paradigmas y los conceptos que están presentes en la intervención social. En este sentido, queda pendiente para futuros estudios analizar en profundidad los roles de sexo-género asignados a las tareas de cuidado en la crianza infantil, especialmente en contextos migratorios y de diversidad cultural.

El otro eje de polarización de los discursos se refiere a aquellas expresiones de agencia infantil donde niños y niñas responderían activamente, ideando mecanismos de protección o expresando 
su presencia en el medio escolar. Referencian como mecanismos la defensa verbal o física para lograr el respeto "por la fuerza" y también el agrupamiento entre pares por nacionalidad, lo que en algunos casos han llamado "defensa corporativa". A través de esta observación contraponen el imaginario de ciertas nacionalidades que califican como sumisas, a las de otras nacionalidades que serían más expresivas e incluso agresivas.

Empezaron a llegar muchos migrantes colombianos y ahí también ha habido problemas, los chicos colombianos son... suelen apiñarse mucho más. Se protegen mucho más (...) se agrupan, que se defienden, molestan a una, reaccionan. Entonces también se ha generado un respeto en base a la fuerza (profesor, escuela pública, Región Metropolitana, Santiago).

Sabido es que vincular ciertas nacionalidades a determinados estereotipos conlleva el riesgo de estigmatización sobre los sujetos. Por lo tanto, es imperioso que los discursos profesionales no reproduzcan juicios de valor, justamente el deber de la investigación social es contribuir a transformar estas apreciaciones. En el actual contexto geopolítico chileno podría llegar a ser ofensivo el nombrar a alguien por su nacionalidad, especialmente si carece de prestigio social, una especie de sinecdóque reduccionista. En los procesos migratorios latinoamericanos existen ciertos imaginarios que operan y contienen una carga simbólica. A veces, las niñas y los niños reproducen los significantes como una forma de burla y porque desconocen los significados y sus matices, pero el mundo adulto lo sabe. En este complejo cruce de lenguajes y etiquetas emerge la agencia infantil migrante, situada en un contexto y territorio particular, es decir, se trata de una agencia imbricada (Güell, 2020).

En las entrevistas se hicieron constantes referencias a conductas transgresoras del orden dentro del centro escolar o de la ley en el ámbito comunitario, al igual que las expresiones de agencia menos expresivas fueron asociadas a una determinada nacionalidad en particular. De acuerdo a estas observaciones, la diferencia entre las conductas calificadas como transgresoras y las menos expresivas sería que las primeras tienden a la deserción del sistema educativo para dedicarse a actividades adultas y las segundas serían pasivas.

Sí, sufren violencia, pero mucho tiene que ver con la relación de los adultos, este tráfico de drogas que te comento, entonces 'es 
hijo de', 'es sobrino de' ... muchos de estos niños están destinados... si ya tienen doce, trece, ya muchos deciden si van a seguir en el tráfico o van a seguir estudiando, porque dejan el colegio de una. Entonces esos niños, que ellos ya saben, son como más osados, son como más puntudos, son como más agrandados, ellos saben que pueden trabajar en el comercio y son niños... [pero] son tratados como adultos (trabajadora social, escuela pública, V Región de Valparaíso, Los Andes).

\section{TABLA 2}

PERCEPCIONES ADULTAS PROFESIONALES COMUNES A LOS MECANISMOS DE AGENCIA INFANTIL "PASIVAS” O “ACTIVAS”

\begin{tabular}{|l|l|}
\hline \multicolumn{1}{|c|}{ Categoría de análisis } & \multicolumn{1}{c|}{ Observación adulta profesional } \\
\hline atribución de causalidad & culturalista \\
& abandono \\
& entorno \\
& marentalización/parentalización \\
\hline rol & reparación \\
& protección \\
& mediación \\
& omisión \\
& incapacidad \\
\hline
\end{tabular}

Fuente: elaboración propia.

\subsection{Explicaciones y roles profesionales}

Para explicar las respuestas infantiles a las situaciones de victimización, algunas y algunos profesionales atribuyen causalidad a distintas variables (Tabla 2). En primer lugar, refieren el abandono familiar, cuya consecuencia sería la marentalización o parentalización, es decir, prácticas de cuidado y crianza desarrolladas por niñas y niños que no deberían realizarlas, desde un punto de vista normativo (Pavez-Soto et al., 2020). Se atribuye a la cultura de ciertos países como la causa de esta supuesta tradición, haciendo una conexión implícita con la conducta violenta. Sin embargo, se podría complejizar esta hipótesis y argüir que los procesos de marentalización/parentalización obedecen no solo a causas culturales, sino también a factores económicos de pobreza en que viven las familias migrantes y las escasas ayudas (redes y 
programas) que reciben para llevar a cabo las tareas del cuidado infantil:

Hemos visto varias familias, principalmente de Bolivia, podría decir, pero, aunque de Colombia y Perú también, donde los hermanos ejercen... las madres son solas y los hermanos mayores ejercen el rol de padres, están parentalizados y entonces tienden a golpear, amenazar y maltratar a sus hermanos menores, como ellos han sido maltratados también, ya sea fuera del país o acá, por su progenitora o por otras personas (psicóloga, institución pública, I región de Tarapacá, Iquique).

Algunas familias migrantes se ubicarían en el lado de la ausencia e incapacidad protectora, que llevaría a niños y niñas hacia los márgenes y las personas profesionales chilenas estarían situadas en el lado de la protección, cuando se tienen los medios y la capacitación para ello.

Niños de corta edad vendiendo, que para ellos es súper normal cosa que para nosotros que somos mucho más protectores, nos llama, así, poderosamente la atención (...). Pero ella cayó en esta red... la denuncia que nos llegó es que efectivamente iba para lo que era el comercio sexual, pero ella decía que solamente vendía artesanía (...) ella no entendía nuestra preocupación, si ella estaba acostumbrada a sobrevivir y a mandarle dinero a sus padres y a sus otros hermanos (trabajadora social, institución pública, Región Metropolitana, Santiago).

En algunos casos, las y los profesionales identificarían que un determinado grupo de padres y madres incentiva a sus hijas e hijos para busquen "el respeto por la fuerza", llegando incluso a intervenir como personas adultas por su propia cuenta, sin esperar intervención institucional, estrategias que promoverían un tipo de agencia negativa:

Sobre todo el colombiano es muy confrontacional. Pero yo creo que tiene que ver con su crianza, con lo que te han dicho en la vida de que ellos se tienen que defender (...) Nosotros hemos tenido grandes conflictos con base a mamás, sobre todo, que han ido a resolver ellas mismas problemas entre los niños y con los papás (trabajadora social, escuela pública, V Región de Valparaíso, Los Andes). 
En segundo lugar, algunas y algunos profesionales atribuyen causalidad al entorno actual y al de sus países de origen, ambos son descritos como intrínsecamente violentos, salvo algunas excepciones donde se reconoce que la exclusión social también afecta a ciertas familias chilenas. Las siguientes citas permiten evidenciar el contraste de observaciones. La primera cita se refiere a un entorno comunitario altamente estigmatizado por los medios de comunicación, donde existen graves problemáticas sociales, que padecen niños y niñas nacionales y extranjeros:

Eso es súper recurrente dado el hecho que ellos vienen con una idea de trabajo y si bien trabajan mucho, hay muchos que se tiran por el lado de las drogas, y puntualmente en Alto Aconcagua hay una gran cantidad de comercio de diferentes estupefacientes. Por lo tanto, las balaceras, los sablazos, los niños lo ven cotidianamente, violencia entre sus padres... que se han matado vecinos migrantes. Pero, así como lo ven los inmigrantes, también lo ven los niños chilenos (trabajadora social, escuela pública, V Región de Valparaíso, Los Andes).

La segunda cita, en cambio, alude al entorno de origen donde residían niños y niñas migrantes, territorios extranjeros que cada profesional asocia con imaginarios que explicarían las conductas infantiles de negociación o defensa:

Me acuerdo que esa directora como que justificaba este tema diciendo que los colombianos venían de la guerra, entonces como que... ellos... estaban preparados (...) como los niños habían aprendido a vivir con la guerra, entonces como que ellos podían negociar en los conflictos (trabajadora social, ONG, Región Metropolitana, Santiago).

El proceso de adaptación sociocultural de las niñas y los niños de cierta nacionalidad sería catalogado con diversos adjetivos, los cuales reflejan los estereotipos que transitan en la sociedad chilena y los respectivos conflictos de aculturación y discriminación que generan:

En el caso de los niños, ellos refieren que muchas veces ellos también pueden ser un poquito más alegres, más dicharacheros (...) en el caso de las niñas las tachan de sueltas y "prostitutas" y todo ese estilo... y en el caso de los chiquillos se les trata también 
mal, porque se dice que son entre comillas alumbrados (...) Y eso a la población local como que molesta... (psicólogo, institución de salud, II región de Antofagasta).

Frente a cualquiera de estas expresiones de agencia, sean del orden expresivo o no expresivo, algunas y algunos profesionales observan su rol en una escala que va desde "reparar" el daño sufrido y "proteger" a niños y niñas de la agresión hasta la "mediación" que intenta conciliar y resolver los conflictos. También hubo observaciones, particularmente sobre los centros escolares, que declaran abiertamente la falta de herramientas para enfrentar las situaciones de agresión (Galaz et al., 2019).

De parte del Ministerio nos falta más apoyo con respecto a cómo tratar y, de alguna manera, cómo utilizar algunas estrategias en la sala de clase para trabajar con niños migrantes. (...) porque también a veces hay niños que su cultura o su forma de ser, de su cultura. En su país como sufren mucha violencia, también vienen con eso y de alguna u otra manera nosotros tenemos que orientarlo y tratar de comprenderlo (...) Entonces falta más apoyo y capacitación a los profesores para trabajar este tema (profesora, liceo público, II región de Antofagasta).

\section{DISCUSIÓN Y CONCLUSIONES}

Los resultados de la presente investigación contribuyen a un análisis sobre las observaciones de los y las adultas profesionales que trabajan con niños y niñas migrantes expuestas a situaciones múltiples de violencia. Revela en primer lugar que, salvo algunas excepciones, se tiende a polarizar las observaciones que hacen sobre las formas que niñas y niños migrantes resisten a las agresiones y, por lo tanto, clasifican sus estrategias de agencia en dos extremos: la sumisión o la agresividad. A su vez, tienden a asociar ambas clasificaciones polares a ciertos estereotipos nacionales, tradiciones culturales e imaginarios de edad o sexo-género, a través de los cuales explican las conductas infantiles y de las familias migrantes. Esta sería una de las principales conclusiones del estudio. No obstante, resulta oportuno preguntarse qué implicancias puede tener para las creencias en torno a la autoeficacia (en el sentido de la agencia 
infantil) y las redes de apoyo a las que pueden acudir las niñas y los niños migrantes cuando están atravesando situaciones de victimización. En segundo lugar y derivado de lo anterior, si acaso es posible concluir en qué medida las condiciones estructurales permiten que niñas y niños migrantes logren transformar las condiciones que les afectan a través de la agencia.

La capacidad de agencia en el campo teórico se aproxima de cierta forma a la idea de resiliencia en el campo de la psicología, modelo que podría utilizarse para el análisis de múltiples factores de riesgo para la polivictimización (Finkelhor et al., 2007). Sin embargo, esta inferencia sería errónea o por lo menos parcial. Por su parte, la resiliencia infantil puede fomentarse cuando las tensiones son manejables y se puede hacer un uso efectivo de las estrategias de afrontamiento (Rutter, 2013 citado en Carr \& Alan, 2016, p. 95), estrategias que son variadas y dependen de diversos factores: personales (biológicos y psicológicos) y contextuales (familiares, parentales y redes sociales) (Carr \& Alan, 2016). Cuestionamos el hecho de que las creencias de los y las profesionales faciliten un uso efectivo de las estrategias psicológicas personales de cada niño o niña, puesto que sus observaciones como personas adultas podrían estar cargadas de prejuicios basados en las nociones de falta, déficit o patología. Una idea que emerge desde la psicología clínica más reciente (Carr \& Alan, 2016) es clave: la estigmatización impacta negativamente la identidad. El lenguaje del trastorno, la discapacidad y el déficit emanados de los manuales de clasificación de trastornos de salud mental, pueden conducir a la autoimagen negativa y baja autoestima, y representa un riesgo para que la persona se observe así misma en función de sus carencias (necesidades e incluso derechos) sin tener en cuenta sus fortalezas, recursos y capacidad de agencia/ resiliencia (Carr \& Alan, 2016, p. 121). De aquí que sea importante que profesionales revisen sus creencias, incluso las aprendidas de los manuales de diagnóstico, evaluando, por ejemplo, si acaso la intervención implica perpetuar las consecuencias y la reincidencia de las situaciones de agresión que han vivido o si por el contrario, representan eventos traumáticos que no son eternos y que no determinan obligadamente un fracaso en sus trayectorias vitales. Los resultados de esta investigación sugieren que la polivictimización en niñas y niños migrantes produce una seria estigmatización que proviene de parte del conjunto de la sociedad receptora, pero se materializa en espacios concretos donde transcurre la vida infantil 
y es ahí, donde emerge la agencia. Queda pendiente para futuros estudios seguir profundizando sobre los mecanismos infantiles para subvertir la vulneración o agresión a través del agenciamiento y cómo se despliegan en el largo plazo las agencias "delgadas", "limitadas", "ambiguas" o "autodestructivas".

La idea de agencia infantil, como hemos visto, cuestiona y reconoce el peso de la estructura social en términos meso y macro sociales: es decir, hasta qué punto un niño o niña migrante logra resistir la violencia del medio en el que se desarrolla o actuar en las estrechas barreras legales y burocráticas de la migración o sus consecuencias mediante estrategias de agencia que no le procuren nuevas agresiones. Entonces, se tensiona el concepto de polivictimización, agencia y los recursos disponibles para hacer frente a la violencia en contextos situados.

Las percepciones profesionales sobre la agencia de niñas y niños migrantes tienden a la polarización. Sitúan, por un lado, a aquellas víctimas de violencia que, clasificados como "sumisas" aceptan o normalizan lo que viven y, por otro lado, aquellos calificados como "agresivos" o demasiado "expresivas" que escapan al paradigma de la infancia moderna. A su vez, ambos polos son asociados a estereotipos de sexo-género, nacionalidad y cultura del país de origen, así como procedencia territorial y estilos marentales y parentales intrínsecamente violentos.

Esta visión reduccionista (sumisión/agresión) reproduce estigmas que suponen trayectorias vitales previamente determinadas. Con lo que podrían perpetuar en sus discursos los efectos de las múltiples agresiones que han vivido. En este sentido, mientras el concepto de polivictimización tiende a centrarse en los aspectos personales de la víctima o aspectos sociales a nivel micro (marentalidad, parentalidad y familia) y se preocupa sobre las futuras consecuencias psico-biológicas de una condición adscrita a las víctimas; la idea de agencia, aunque tampoco del todo acabada, se abstiene de adscribir sentencias permanentes en términos de los efectos de un trauma. Más bien aporta una perspectiva meso y macro social, que destaca las condiciones del entorno social intermedio y también aquellas estructurales de los países de recepción, que condicionan la expresión de la agencia, desde aquellas más "gruesas" o con mayor margen de acción hasta aquellas más "delgadas" o con posibilidades restringidas que pueden profundizar la vulneración de sus derechos y quedar sujetas a la intervención adulta. De todas formas, sigue 
siendo un desafío teórico y empírico la discusión por el modo en que la agencia infantil puede incidir en la estructura social y viceversa, sin dejar de reconocer la autonomía de cada elemento (Archer, 2009) y, por lo tanto, explicar como serían posibles los cambios sociales.

\section{REFERENCIAS BIBLIOGRÁFICAS}

Archer, M. (2009). Teoría social realista: En enfoque morfogenético. Ediciones Universidad Alberto Hurtado.

Atkinson-Sheppard, S. (2017). Street Children and 'Protective Agency': Exploring Young People's Involvement in Organised Crime in Dhaka, Bangladesh. Childhood, 24(3),416-29.

Bordonaro, L. y Payne, R. (2012). Ambiguous agency: critical perspectives on social interventions with children and youth in Africa. Children's Geographies, 10(4),365-72.

Caneva, E. (2015). Children's agency and migration: Constructing kinship in Latin American and East European families living in Italy. Childhood, 22(2),278-92.

Carr \& Alan (2016). The handbook of child and adolescent clinical psychology: a contextual approach. 3 rd ed. Routledge.

Cedeño, L., Martínez-Arias, R. y Bueno, J. (2014). Latino immigrant students in Spain: Potential poly-victims and school underachievers. Electronic Journal of Research in Educational Psychology, 12(3),803-34.

Cerón, L., Pérez Alvarado, M. y Poblete, R. (2017). Percepciones Docentes en torno a la Presencia de Niños y Niñas Migrantes en Escuelas de Santiago: Retos y Desafíos para la Inclusión. Revista latinoamericana de educación inclusiva, 11(2),233-46.

Chen, X., Liang, N. y Ostertag, S. (2017). Victimization of Children Left Behind in Rural China. Journal of Research in Crime and Delinquency, (4),515-543.

Consejo Nacional de la Infancia (2018). Análisis Multivariable de Estudio Polivictimización en Niños, Niñas y Adolescentes realizado por la Pontificia Universidad Católica de Chile. Santiago, Chile.

Cortez, C., Pereda, N. y Flores, J. (2017). Estudios sobre violencia interpersonal contra la infancia y la adolescencia en Chile: una revisión. Interciencia: Revista de ciencia y tecnología de América, 42(5),277-85.

Cortez, C., Flores, J., Pereda,N. y Guerra, C. (2019). Victimización y polivictimización en niños, niñas y adolescentes aymara y su relación con sintomatología post-traumática. Interciencia: Revista de ciencia y tecnología de América, 44(4),229-35.

Dreby, J. (2007). Children and Power in Mexican Transnational Families. Journal of Marriage and Family, 69(4),1050-64. 
Dreby, J. (2015). U.S. Immigration Policy and Family Separation: The Consequences for Children's Well-Being. Social Science \& Medicine, 132, 245-51.

Dreby, J. y Adkins, T. (2012). The Strength of Family Ties: How US Migration Shapes Children's Ideas of Family. Childhood, 19(2),169-87.

Esser, F., Baader, M., Betz, T. y Hungerland, B. (2016). Reconceptualising Agency and Childhood: New Perspectives in Childhood Studies. Routledge.

Finkelhor, D., Ormrod, R., \& Turner, H. (2007). Poly-Victimization: A Neglected Component in Child Victimization. Child Abuse \& Neglect, 31(1),7-26.

Finkelhor, D., Ormrod, R., \& Turner, H. (2009). Lifetime Assessment of PolyVictimization in a National Sample of Children and Youth. Child Abuse \& Neglect, 33(7),403-11.

Finkelhor, D., Turner, H., Hamby, S. y Ormrod, R. (2011). Polyvictimization: Children's Exposure to Multiple Types of Violence, Crime, and Abuse. $\mathrm{Na}$ tional Survey of Children's Exposure to Violence.

Ford, J., \& Delker, B. (2018). Polyvictimization in childhood and its adverse impacts across the lifespan: Introduction to the special issue. Journal of Trauma \& Dissociation, 19(3),275-88.

Gaitán, L., \& Liebel., M. (2011). Ciudadanía y derechos de participación de los niños. Edición: 1. Madrid: Sintesis.

Galaz, C., Pavez-Soto, I., Alvarez, C. y Hedrera, L. (2019). Polivictimización y agencia de niños y niñas migrantes en Chile desde una mirada interseccional. Athenea Digital. Revista de pensamiento e investigación social 19(2),2447-2447.

Garnier, P. (2015). L“'agency” des enfants. Projet scientifique et politique des "childhood studies". Education et societes, 36(2),159-73.

Gigengack, R. (2008). Critical Omissions: How Street Children Studies Can Address Self-Destructive Agency. Research with Children: Perspectives and Practices: Second Edition 205-19.

Gonçalves, M. y Matos, M. (2020). Mental health of multiple victimized immigrant women in Portugal: Does resilience make a difference? Journal of Human Behavior in the Social Environment, 30(3),353-68.

Güell, B., Arrasate, M. y Solé, A. (2020). Visibilizando estrategias de agencia y empoderamiento económico: El caso de las mujeres de origen pakistaní en Barcelona. Migraciones. Publicación del Instituto Universitario de Estudios sobre Migraciones, (48), 51-78. https://doi.org/10.14422/mig. i48y2020.003

Guerra, C., Inostroza, R., Villegas, J., Villalobos, L., Pinto-Cortez, C., Guerra, C. y Inostroza, R., (2017). Polivictimización y sintomatología postraumática: el rol del apoyo social y la autoeficacia. Revista de psicología (Santiago), 26(2),66-75.

Haring, U., Sorin, R. y Caltabiano, N. (2019). Reflecting on Childhood and Child Agency in History. Palgrave Communications, 5(1),1-9.

Honwana, A. (2005). Child Soldiers in Africa. Pennsylvania, Philadelphia, USA: University of Pennsylvania Press. 
Hongwei, H., Zhu, X. y Li, Y., (2018). The association and mediating mechanism between poverty and poly-victimization of left-behind children in rural China. Children and Youth Services Review, 91, 22-29.

Huijsmans, R. (2012). Beyond Compartmentalization: A Relational Approach towards Agency and Vulnerability of Young Migrants. New Directions for Child and Adolescent Development, 2012(136),29-45.

INE, y DEM. (2020). Estimación de personas extranjeras residentes habituales en Chile al 31 de diciembre 2019. Informe Técnico. Instituto Nacional de Estadísticas y Departamento de Extranjería y Migración.

Instituto Nacional de Estadísticas. (2018). Características de la inmigración internacional en Chile, Censo 2017. Santiago de Chile: Instituto Nacional de Estadística, Chile.

James, A. y James, A. (2012). Key Concepts in Childhood Studies. Second Edition. London.

James, A. y Prout, A. (1997). Constructing and Reconstructing Childhood: Contemporary Issues in the Sociological Study of Childhood. 2 edition. London ; Washington, D.C: Routledge.

Jenks, C. (1996). Childhood. London ; New York: Routledge.

Klocker, N. (2007). An Example of ‘Thin' Agency. Child Domestic Workers in Tanzania. En Global Perspectives on Rural Childhood and Youth: Young Rural Lives (pp. 83-94), editado por R. Panelli, S. Punch, y E. Robson. Routledge.

Llobet, V. (2012). Políticas sociales y ciudadanía: Diálogos entre la teoría feminista y el campo de estudios de infancia. Frontera norte, 24(48),2.

Margarit, D. y Bijit, K. (2014). Barrios y población inmigrantes: el caso de la comuna de Santiago. Revista INVI, 29(81),19-77.

Mayall, B. (2000). The Sociology of Childhood in Relation to Children's Rights. The International Journal of Children's Rights, 8(3),243-59.

Mayall, B. (2002). Towards A Sociology For Childhood: Thinking from Children's Lives. Buckingham ; Philadelphia: Open University Press.

Ministerio de Desarrollo Social. (2017). Inmigrantes Síntesis de resultados CASEN 2017. Subsecretaría de Evaluación Social.

Ministerio de Desarrollo Social. 2020. «Base de datos encuesta Casen 2017». Observatorio Social. Recuperado 30 de marzo de 2020 (http://observatorio. ministeriodesarrollosocial.gob.cl).

Moscoso, M. (2013). Biografía para uso de los pájaros: memoria, infancia y migración. 1. a ed. Quito, Ecuador: IAEN.

Parella, S. y Marcela T. (2015). Las regiones fronterizas para el estudio de la migración y la circulación. Un análisis a partir de dos casos ilustrativos. Pp. 173-206 en Las fronteras del transnacionalismo: límites y desbordes de la experiencia migrante en el centro y norte de Chile. Santiago, Chile: Ocholibros.

Parsons, T. (1968). La estructura de la acción social. Ediciones Guadarrama.

Parsons, T. (1999). El sistema social. Alianza. 
Pavez-Soto, I., Galaz, C., Poblete-Godoy, D., Acuña, V. y Sepúlveda, N. (2020). Horizontes de la intervención social con infancia migrante en Chile. Revista Rumbos TS. Un Espacio Crítico Para La Reflexión En Ciencias Sociales, (23), 9-40. https://doi.org/10.51188/rrts.num23.403

Pavez-Soto, I., Ortiz-López, J., Sepúlveda, N., Jara, P. y Olguín, C. (2019). Racialización de la niñez migrante haitiana en escuelas de Chile. Interciencia: Revista de ciencia y tecnología de América, 44(7),414-20.

Pavez-Soto, I. (2011). Migración infantil: rupturas generacionales y de género. Las niñas peruanas en Barcelona y Santiago de Chile. Ph.D. Thesis, Universitat Autònoma de Barcelona.

Pavez-Soto, I. (2018). Violencias contra las infancias migrantes en Santiago de Chile: resistencias, agencia y actores. Migraciones Internacionales, 9(35), 155-84.

Pavez-Soto, I. y Sepúlveda, N. (2019). Concepto de agencia en los estudios de infancia. Una revisión teórica. Sociedad e Infancias, 3, 193-210.

Pereda, N., \& Gallardo-Pujol, D. (2014). One Hit Makes the Difference: The Role of Polyvictimization in Childhood in Lifetime Revictimization on a Southern European Sample. Violence and Victims, 29(2),217-31.

Piras, G. (2016). Emociones y migración: Las vivencias emocionales de las hijas y los hijos que se quedan en origen. Psicoperspectivas. Individuo y Sociedad, 15(3),67-77.

Poblete, R. y Galaz, C. (2017). «Aperturas y cierres para la inclusión educativa de niños/as migrantes en Chile». Estudios pedagógicos (Valdivia), 43(3),239-57.

Qvortrup, J. (1994). Formas de acercarse a las vidas y actividades de los niños. Madrid: Ministerio de Asuntos Sociales.

Riedemann, A. y Stefoni, C. (2015). Sobre el racismo, su negación, y las consecuencias para una educación anti-racista en la enseñanza secundaria chilena. Polis. Revista Latinoamericana (42).

Ródenas, B. (2017). Hogares conyugales y redes sociales: la agencia de las senegalesas migrantes. Migraciones. Publicación del Instituto Universitario de Estudios sobre Migraciones, (41), 29-53. https://doi.org/10.14422/mig. i41.y2017.002

Rübner, C. (2016). 'The problem is that I don't know' - Agency and life projects of transnational migrant children and young people in England and Spain. Childhood, 24(1),21-35.

Segura, A., Magallón-Neri, E., Soler, L. Kirchner, T. y Forns, M. (2015). Polyvictimization and Its Effects On Mental Health Problems Among Immigrant and Native Adolescents in Catalonia. European Psychiatry, 30,858 .

Sellenet, C. (2007). Familles et enfants sous haute surveillance. Le Journal des psychologues, 250(7),67-70.

Sime, D. y Fox, R. (2015). Home abroad: Eastern European children's family and peer relationships after migration. Childhood, 22(3),377-93. 
Strandell, H. (2010). From Structure-Action to Politics of Childhood: Sociological Childhood Research in Finland. Current Sociology.

Strauss, A. y Corbin, J. (2002). Bases de la investigación cualitativa: técnicas y procedimientos para desarrollar la teoría fundamentada. Universidad de Antioquia.

Thompson, A., Torres, R., Swanson, K., Blue, S. y Hernández, O. (2019). Reconceptualising agency in migrant children from Central America and Mexico. Journal of Ethnic and Migration Studies, 45(2),235-52.

Tijoux-Merino, M. (2013). Niños(as) marcados por la inmigración peruana: estigma, sufrimientos, resistencias. Convergencia Revista de Ciencias Sociales (61).

Ungruhe, C. (2019). Beyond Agency's Limits. “Street Children's” Mobilities in Southern Ghana. Cadernos de Estudos Africanos, (37),41-61.

UNICEF. 2012. $4^{\circ}$ estudio de maltrato infantil en Chile. Análisis comparativo 1994-2000-2006-2012. Santiago, Chile: UNICEF.

Wihstutz, A. (2020). 'I can't sit here and cry with you, I have to play'. Strategies by very young children in refugee collective accommodation. European Early Childhood Education Research Journal, 28(1), 115-128, DOI: 10.1080/1350293X.2020.1707367 
